\title{
Quality of Life and Its Contributing Factors among Breast Cancer Patients after Intensive Treatment
}

\author{
Tran Thua Nguyen ${ }^{1)}$, Pham Nguyen Tuong ${ }^{2)}$, Phan Thi Do Quyen ${ }^{2)}$, Nguyen Huu Son ${ }^{3)}$ \\ ${ }^{1)}$ Department of General Internal Medicine and Geriatrics, Hue Central Hospital, Hue city, Vietnam \\ ${ }^{2)}$ Oncology Center, Hue Central Hospital, Hue city, Vietnam \\ ${ }^{3)}$ Pediatric Center, Hue Central Hospital, Hue city, Vietnam
}

\begin{abstract}
Objective: The survey aims to explore the quality of life and its contributing factors among breast cancer patients who had been treated at the hospital and returned for follow-up care and/or for taking hormonal therapy.

Methods: This cross-sectional study deployed questionnaire-based interviews with 115 patients when they came to the hospital for follow-up care and/or taking hormonal therapy at the Oncology Center of Hue Central Hospital (Vietnam) from April to May 2019. Collected data were analyzed using SPSS software 21 for Windows.

Results: The mean age of our study was 53.2 years. Among the total respondents, $88.7 \%$ of patients had hormone receptorpositive breast cancer. Most respondents reported experiencing symptoms that decreased their health-related quality of life, such as dry mouth, appetite changes, fatigue, arm pain, and limited mobility in ipsilateral arm. The majority felt insecure because of their asymmetric and less good-looking post-mastectomy body image. Most of them were no longer interested in sexual activities. A large number of respondents turned to complementary therapies at home, which often incurred considerable costs.

Conclusion: This survey contributes to the understanding of patients' suffering symptoms, their concerns and anxiety, and distress during outpatient care/ clinic. It also provides baseline information on their thoughts and preferences for complementary therapies. Altogether the study carries important implications for clinicians to plan more effective treatment and care for breast cancer patients.
\end{abstract}

Keywords: quality of life, breast cancer, burden of cancer

(Received September 9, 2020; Accepted September 15, 2020)

\section{Background}

Breast cancer is the most common cancer among women all over the world ${ }^{1,2)}$. In Vietnam, according to cancer registry data in 2010, breast cancer ranked first with an average age-standardized rate of 29.9/100,000 people. It is estimated that by 2020 , this rate will rise to 38.1/100,000 people $^{3)}$. Medical innovations, especially in the field of breast cancer treatment and the combination of multi-modal and multi-disciplinary treatments, have promoted the survival rate of cancer ${ }^{4,5)}$. Therefore, the additional survival time after treatment and the overall survival rate of patients has been increasing. More attention is now paid to the quality of life of the patients in addition to treatment effectiveness ${ }^{6,7)}$. Cancer patients, women living with breast cancer in particular, might experience a different state of emotions after being di-

Corresponding Author: Dr. Pham Nguyen Tuong, MD,PhD. Oncology Center, Hue Central Hospital, 16 Le Loi street, Hue, Vietnam. Tel: +849913493432. E-mail: phamnguyentuongubhue@gmail.com agnosed with cancer. They might be shocked, anxious, worried, and desperate ${ }^{8,9)}$. After accepting their diagnosis, they start to suffer physical pain resulting from their treatment, such as surgery, chemotherapy, and radiation. After having undergone radical treatment, patients undergo some of the unexpected treatment side effects over a long time, and sometimes lots of obsessions are generated for the rest of their lives. All these things affect the physiology of the patient. A typical profile of a breast cancer patient is a woman who has a part or the whole breast with the tumor removed and will experience adjuvant treatments, such as chemotherapy, radiation, and endocrine therapies. These therapies can all exert longlasting effects on the body, which can all have long-lasting effects on the body- female hormones and functional activities of the ipsilateral arm will decrease, as well as the patisent's sexual life ${ }^{5)}$. Since Asian women, as well as Vietnamese ones, are often shy when being inquired about sexual-related topics, researches on this issue still remain limited.

We conducted this research to explore factors affecting the quality of life, including the sexual life of 
breast cancer patients who have undergone treatment and were, at the time of the study, being monitored during follow-up care or taking hormonal medicine at an oncology center in Vietnam. Besides, we also explored the situation regarding the use of traditional therapies traditional Chinese medicine, herbs, or folk remedies that are being rumored by patients to treat cancer, and functional foods that are also widely used. A number of trends in extreme diets had also being surveyed and examined. These data both help us understand the patients' life and the knowledge they gained from several sources so that each clinician can have a better understanding of the patient's concerns AND aid in doctors' delivery of more comprehensive treatment to minimize the harmful effects of some prescriptions. Besides, we might also see an increase in the patient's economic burden due to their belief in misleading advertisements of some complementary foods.

\section{Materials and Methods}

\section{Study population}

This study collected data from 115 patients who came to the clinic for examination at the Oncology Center at Hue Central Hospital and met the following criteria: (1) Diagnosed with breast cancer, having undergone surgery and previously received inpatient treatment (chemotherapy, radiation therapy if indicated), currently only being monitored and/ or received hormonal therapy at the clinic between April 2019 and May 2019. (2) Having the ability to listen, understand, and talk. (3) Being explained about the survey and voluntarily taking part in interviews. Participation in the study did not affect the patient's treatment or examination plan.

The exclusion criteria were: (1) Patient refused to participate in the survey. (2) Breast cancer patients were receiving inpatient treatment at the time of the study.

\section{Data collection}

A questionnaire based on the Vietnamese version of EORTC QLQ BR23, a tool to inquire about the quality of life developed by the European Cancer Research and Treatment Organization (The European Organization for Research and Treatment of Cancer), was deployed ${ }^{10)}$. This questionnaire was adapted to be more culturally appropriate to the contexts of patients at the Oncology Center Hue Central Hospital.

\section{Statistical analysis}

Data was recorded by face-to-face questionnaires, gathered and processed using SPSS 21.0 for Windows software.

Main evaluation criteria: Factors that cause a decrease in a patient's quality of life, her sexual life, diets, and home care.

\section{Results}

\section{Baseline characteristics}

Female patients who have been diagnosed with breast cancer and had surgery may have undergone chemotherapy, radiation, or hormonal therapy. The mean age was 53.22 years.

Breast tumor position: on the right (59 patients, accounting for $51.3 \%$ ), and on the left (56 patients, $48.7 \%$ ). Metastases were not seen in 100 patients (87\%), while the remaining 15 patients have had relapses or metastases and had been treated second line (chemotherapy or hormonotherapy). The number of patients with positive hormone receptors was $102(88.7 \%)$. The number of patients with Her2neu positive was 31 (27\% of the total samples).

13 of these 115 patients had breast-conserving surgery, meaning they did not have their breasts completely removed. These patients still retained their breasts.

The number of patients who received intensive treatments including chemotherapy and radiotherapy was $80(69.6 \%)$ and $57(49.6 \%)$ respectively. Among patients with positive hormone receptors, 34 patients were treated with Tamoxifen (with or without ovarian function suppression), the remaining were treated with Aromatase Inhibitors with active ingredients Anastrozole, Letrozole, or Exemestane.

Table 1 shows the symptoms that decrease a patient's quality of life. The symptoms of dry mouth, loss of appetite could be the prolonged unwanted effects of previous treatments such as chemotherapy or hormonal drugs. Another potential cause for these symptoms is psychological anxiety, stress, or illness. Besides, strict diets can contribute to these symptoms. Bodily pain was a common symptom among the surveyed patients. This might be due to previous treatments such as adjuvant chemotherapy or hormonal drugs, because the chemotherapy process temporarily suppresses the ovaries, causing the hormonal decline in young patients, causing problems such as muscle aches, and hot flush. Anorexia and insomnia were also common symptoms among breast cancer patients who were examined at the clinic. They might be some of the biggest reasons attributed to the patient's anxiety and their strict diet, resulting in a decrease in

Table 1 Symptoms that decrease a patient's quality of life

\begin{tabular}{l|c|c|c|c}
\hline \multirow{2}{*}{ Symptoms } & \multicolumn{4}{|c}{ Extent, n (\%) } \\
\cline { 2 - 5 } & Not at all & Rarely & Often & Very often \\
\hline Dry mouth & $54(47.0 \%)$ & $41(35.7 \%)$ & $15(13.0 \%)$ & $5(4.3 \%)$ \\
\hline $\begin{array}{l}\text { Loss of appetite com- } \\
\text { pared to pre-treatment } \\
\text { period }\end{array}$ & $84(73.0 \%)$ & $18(15.7 \%)$ & $12(10.4 \%)$ & $1(0.9 \%)$ \\
\hline Whole body pain & $55(47.8 \%)$ & $48(41.7 \%)$ & $12(10.4 \%)$ & $0(0.0 \%)$ \\
\hline Hot flush & $31(27.0 \%)$ & $45(39.1 \%)$ & $23(20.0 \%)$ & $16(13.9 \%)$ \\
\hline Loss of appetite & $80(69.6 \%)$ & $25(21.7 \%)$ & $7(6.1 \%)$ & $5(4.3 \%)$ \\
\hline Insomnia & $47(40.9 \%)$ & $45(39.1 \%)$ & $15(13.0 \%)$ & $8(7.0 \%)$ \\
\hline
\end{tabular}


Table 2 Possible long-term side-effects of mastectomy

\begin{tabular}{l|c|c|c|c}
\hline \multirow{2}{*}{ Symptoms } & \multicolumn{4}{|c}{ Extent, $(\%)$} \\
\cline { 2 - 5 } & Not at all & Rarely & Often & Very often \\
\hline Pain in arm & $32(27.8 \%)$ & $52(45.2 \%)$ & $21(18.3 \%)$ & $10(8.7 \%)$ \\
\hline Swollen arm & $88(76.5 \%)$ & $21(18.3 \%)$ & $4(3.5 \%)$ & $2(1.7 \%)$ \\
\hline $\begin{array}{l}\text { Difficulty when mov- } \\
\text { ing arms }\end{array}$ & $66(57.4 \%)$ & $30(26.1 \%)$ & $15(13.0 \%)$ & $4(3.5 \%)$ \\
\hline $\begin{array}{l}\text { Oversensitiveness at } \\
\text { affected breast }\end{array}$ & $29(25.2 \%)$ & $66(57.4 \%)$ & $15(13 \%)$ & $5(4.3 \%)$ \\
\hline
\end{tabular}

their quality of life.

Most breast cancer patients who had undergone a radical mastectomy in this survey complained of feeling pain in the breast and the ipsilateral arm, particularly the armpit and the inner parts of the arm. Some patients might have mild edema of the arms and the breast. $75 \%$ of patients who underwent radical mastectomy had a feeling of paresthesia in the area of the removed breast. $34 \%$ of patients had difficulty moving the arm that is on the same side where the breast has been removed. These symptoms caused discomfort in the daily life of the patient (Table 2).

Breasts represent a woman. Most women feel confident in their shape when they have beautiful and complete breasts. Therefore, the removal of one breast, the imbalance with the large old incision and the ugly scarring after radical mastectomy have caused significant effects to most women with breast cancer. Some patients did not even dare to look at themselves in the mirror. A common answer was that they were uncomfortable of looking at themselves. The proportion of those who were uncomfortable was $48.7 \%$ (56 patients), while 32 patients (27.8\%) were rarely uncomfortable but at a lesser extent. Less than $25 \%$ of patients did not feel disatified looking at themselves in the mirror (Table 3).

Among 115 surveyed patients, although there were only 15 patients (13\%) having low curable capability since they were classified as advanced-stage with cancer recurrence or metastases, the number of patients worrying about their health was particularly high, 87.8\% (101 patients). In particular, the number of patients who worried a lot was 41 , accounting for $35.7 \%$. Thereby, it was shown that cancer patients, despite being at stable treatment state and having high chances of curability, still reported psychological anxiety and lived in constant fear.

All patients were outpatients who came to the clinic to be tested and monitored during re-examination, or to take hormonal drugs which are reported as having insignificant side effects. Only 15 patients developed a new recurrence after step 1 . However, most of the respondents thought that they felt sick. That was, to those patients' thinking, they were still unable to live a normal life. The percentage of patients who always felt sick at high and very high frequency was $26.1 \%$ (20 patients). Only 38 patients did not report their feeling of sickness.
Table 3 Insecurity due to post-mastectomy body disfigurement

\begin{tabular}{l|c|c|c|c}
\hline \multirow{2}{*}{ Symptoms } & \multicolumn{4}{|c}{ Extent, n (\%) } \\
\cline { 2 - 5 } & Not at all & Rarely & Often & Very often \\
\hline Feeling ugly & $28(24.3 \%)$ & $40(34.8 \%)$ & $35(30.4 \%)$ & $12(10.4 \%)$ \\
\hline Decreased femininity & $29(25.2 \%)$ & $40(34.8 \%)$ & $40(34.8 \%)$ & $6(5.2 \%)$ \\
\hline $\begin{array}{l}\text { Feeling difficult look- } \\
\text { ing at one's self in the } \\
\text { mirror }\end{array}$ & $27(23.5 \%)$ & $32(27.8 \%)$ & $44(38.3 \%)$ & $12(10.4 \%)$ \\
$\begin{array}{l}\text { Dissatisfied with the } \\
\text { body }\end{array}$ & $26(22.6 \%)$ & $44(38.3 \%)$ & $7(6.1 \%)$ & $1(0.9 \%)$ \\
\hline
\end{tabular}

\section{Sexual drive and activity}

$81 \%$ of the respondents said that they were not interested in sexual activity. Meanwhile, $58 \%$ of patients reported they no longer engaged in any sexual activity. The reason for their inactivity was: no interest, pain, fatigue. Notably, 15\% (17 patients) said they were afraid that having sex could increase the risk of cancer recurrence.

\section{The use of complementary foods at home}

When patients are at home, the use of traditional or Chinese medicines, herbs and complementary foods is quite common. Medications vary from patient to patient and change over time. In this survey, we recorded that 74 patients out of 115 patients, accounting for $64.3 \%$ of respondents, were able to use other types of herbal medicines, traditional/ Chinese medicines at home. Many patients used many types at the same time and did not consult with their oncologists or traditional medicine doctors. Most of them acquired information from acquaintances, other patients or online advertisements. The most common types that were currently in use were: Papaya leaves/ flowers. Most information about this type of treatment was transmitted among patients, their relatives and friends. In addition, a number of other drugs were also used extensively for the purpose of cancer treatment such as: Radiation black, ventricular, Turmeric starch, and seaweed pill.

The average amount of money that patients spent on these treatments was about VND 340,000 per month.

\section{Special diets and abstinence}

More than $50 \%$ of the respondents said they abstained from eating a diet that was full of meat, sugars, milk, and eggs, etc. Their diets lacked the key nutrients to maintain a healthy body. This was also one of the possible causes of anorexia. This strict abstinence significantly reduced the patient's quality of life. Among the interviewees, there were 3 cases following the sesame brown rice diet (also known as Osawa macrobiotic diet). This was a diet that caused severe deficiency and renders the lives of patients cramped and struggling. However, patients still wanted to follow this diet in the hope that their cancer would not recur (Table 4). 
Table 4 Abstained food

\begin{tabular}{l|c}
\hline \multicolumn{1}{c|}{ Abstained food } & $\mathrm{n}(\%)$ \\
\hline Red meat & $21(18.3 \%)$ \\
\hline Dairy products including milk & $2(1.7 \%)$ \\
\hline All nutrition (protein/sugar/milk) & $67(58.3 \%)$ \\
\hline Sugar & $7(6.1 \%)$ \\
\hline Permanent vegetarian & $2(1.7 \%)$ \\
\hline Sesame brown rice diet & $3(2.6 \%)$ \\
\hline
\end{tabular}

\section{Dicussion}

We found similar findings in this study to those in some other studies in the world. For instance, the ratio of tumor location was equal between the two sides. The proportion of patients with positive hormone receptors usually accounted for two-thirds of all breast cancer patients. The rate of positive Her2neu receptors accounted for about $25 \%$ of breast cancer patients ${ }^{11,12)}$. Patients who had been treated with methods such as surgery, chemotherapy/radiation, were turned to follow-up monitoring or outpatient medications. However, the comprehensive care process for cancer patients requires regular examination and monitoring, not only during the period of inpatient care. Some big problems will greatly reduce outpatients' quality of life if they are not timely addressed. Firstly, symptoms may persist after previous treatment such as body aches, dry mouth, and loss of appetite. The incidence of these symptoms in our patients was over $30 \%$. This rate corresponded to those reported in a number of studies conducted in Western Europe among postradical-mastectomy breast cancer patients such as research by Johannsen et al. ${ }^{13)}$. After radical mastectomy, more than $20 \%$ of patients experienced feeling of pain in the old incision area and the ipsilateral arm. The proportion of those with the feeling of moderate to significant pain and had difficulty moving their arms were $60 \%$. and $42.6 \%$, respectively. These are quite common symptoms after radical mastectomy. These ratios were similar to the studies of Johannsen et al, the study of Leysen et al. ${ }^{14)}$. From these data, we could see a notable issue that needed attention for patients after radical mastectomy. This imposed an urgent need to improve the long-term quality of life for breast cancer patients after intensive treatment.

An another important issue that has not thoroughly researched is the disastification towards the patient's physical appearance after a radical mastectomy. In our study, most patients felt that they became less pretty, experienced reduced femininity and felt scared when they looked at themselves in the mirror. This was one of the factors causing significant psychological pressure for breast cancer patients after surgery. In the interviewed patients, both young or old patients were still very concerned about their body shape. The rate of feeling ugly was $75.2 \%$ ( 87 patients). 88 (76.5\%) patients found it dif- ficult to look at themselves in the mirror. The number of patients who were dissatisfied with their own body was 89 patients, accounting for $77.3 \%$. This had also been observed in some studies conducted among Asian patients such as those in Taiwan by Chie et al. ${ }^{15)}$ or research in Jordan by Abu-Helalha et al. ${ }^{16)}$. From the above figures, it was suggested that we need to provide reconstructive breast surgery to patients who underwent radical mastectomy and had completed treatment as part of their comprehensive care plan for a breast cancer patient. Such treatment would significantly improve the quality of life and make the treatment of breast cancer better, contributing to restoring the physical and mental health of the patient.

An indispensable factor for a good quality of life is the sex life of a breast cancer patient after treatment. For patients who had a mastectomy, their physical disfigurement remarkably affects this aspect. Given the picture regarding sex life activity among surveyed patient group, the quality of life of the patients will be significantly reduced. In addition, due to the cultural and behavioral characteristics of Asian people, the sex life of patients have not been paid attention, preventing the provision of timely interventions. This conclusion was also found in a number of studies in other Asian countries like Taiwan or Jordan ${ }^{16)}$. In addition, a number of other factors also result in a decrease in the quality of a patient's sex life, such as the use of hormonal drugs and the side effects of the drug on the patient's reproductive system, anxiety and worry about illness, feeling sick, and the health deterioration after treatment, or strict diet. All of these factors combine and leave certain influence on a patient's sex life.

A major problem affecting the quality of life and financial situation of patients is their common use of alternative treatments. That is a prevalent situation in most Asian patients, which can also easily be found in a number of studies conducted in Korean and Chinese settings.

\section{Conclusion}

Understanding factors affecting the patient's quality of life including physical, mental and sexual issues, trend of using replacement therapies will aid clinicians in the process of treatment planning and providing advice to patients before, during and after the intensive treatment, as well as during maintenance medication. With a specific, accurate and appropriate plan for each patient, the chance of success in our treatment will increase, which consequently promote the likelihood of curability and minimize possible complications during treatment. 


\section{References}

1) Coughlin SS. Epidemiology of Breast Cancer in Women. Adv Exp Med Biol. 2019. 1152: 9-29.

2) Youn HJ, Han W. A Review of the Epidemiology of Breast Cancer in Asia: Focus on Risk Factors. Asian Pac J Cancer Prev. 2020. 21 : 867-880.

3) Pham T, Bui L, Kim G, Hoang D, Tran T, Hoang M. Cancers in Vietnam-Burden and Control Efforts: A Narrative Scoping Review. Cancer Control. 2019. 26: 1073274819863802.

4) Charmsaz S, Prencipe M, Kiely M, Pidgeon GP, Collins DM Innovative Technologies Changing Cancer Treatment. Cancers (Basel). 2018. 10(6): 208.

5) Moo TA, Sanford R, Dang C, Morrow M. Overview of Breast Cancer Therapy. PET Clin. 2018. 13: 339-354.

6) Paraskevi T. Quality of life outcomes in patients with breast cancer Oncol Rev. 2012. 6: e2.

7) Shafaie FS, Mirghafourvand M, Amirzehni J. Predictors of Quality of Life in Patients with Breast Cancer. Indian J Palliat Care. 2019. 25: 73-78.

8) Baqutayan SM. The effect of anxiety on breast cancer patients Indian J Psychol Med. 2012. 34: 119-23.

9) Davis C. Psychosocial needs of women with breast cancer: how can social workers make a difference? Health Soc Work. 2004. 29: 330-4.

10) Sprangers MA, Groenvold M, Arraras JI, Franklin J, te Velde A, Muller M, Franzini L,Williams A, de Haes HC, Hopwood P, Cull A, Aaronson NK. The European Organization for Research and Treatment of Cancer breast cancer-specific quality-of-life questionnaire module: first results from a three-country field study. J Clin Oncol. 1996. 14: 2756-68.
11) Dai X, Xiang L, Li T, Bai Z. Cancer Hallmarks, Biomarkers and Breast Cancer Molecular Subtypes. J Cancer. 2016. 7: 1281-94.

12) Goddard KA, Weinmann S, Richert-Boe K, Chen C, Bulkley J, Wax C. HER2 evaluation and its impact on breast cancer treatment decisions. Public Health Genomics. 2012. 15: 1-10.

13) Johannsen M, Christensen S, Zachariae R, Jensen AB. Sociodemographic, treatment-related, and health behavioral predictors of persistent pain 15 months and 7-9 years after surgery: a nationwide prospective study of women treated for primary breast cancer. Breast Cancer Res Treat. 2015. 152: 645-658.

14) Leysen L, Beckwee D, Nijs J, Pas R, Bilterys T, Vermeir S, Adriaenssens N. Risk factors of pain in breast cancer survivors: a systematic review and meta-analysis. Support Care Cancer. 2017. 25: 3607-3643.

15) Chie WC, Chang KJ, Huang CS, Kuo WH. Quality of life of breast cancer patients in Taiwan: validation of the Taiwan Chinese version of the EORTC QLQ-C30 and EORTC QLQ-BR23. PsychoOncology. 2003. 12: 729-735.

16) Abu-Helalah M, Al-Hanaqta M, Alshraideh H, Abdulbaqi N, Hijazeen J. Quality of life and psychological well-being of breast cancer survivors in Jordan. Asian Pac J Cancer Prev. 2014. 15: 5927-36. 